Check for updates

Cite this: RSC Adv., 2019, 9, 33617

\title{
Melanin-mimetic multicolor and low-toxicity hair dye $\uparrow$
}

\author{
Yingying Dong, $\dot{t}^{\mathrm{a}}$ Yan Qiu,,$_{+}^{\mathrm{b}}$ Die Gao, ${ }^{\mathrm{b}}$ Kailian Zhang, ${ }^{\mathrm{b}}$ Kai Zhou, ${ }^{\mathrm{c}}$ Honggang Yin, ${ }^{\mathrm{b}}$ \\ Gaoyi Yi, ${ }^{b}$ Jun Li, ${ }^{* a}$ Zhining Xia (D) ${ }^{d}$ and Qifeng Fu (D)*b
}

Most commercial permanent hair dyeing technologies are based on the oxidative process of $p$ phenylenediamine and its derivative materials. However, concerns about their toxicological issues have been raised throughout the years. Herein, we report an innovative surface coloration strategy for fabricating melanin-mimetic multicolor and low-toxicity hair dyes through sodium periodate-induced rapid deposition of eumelanin-like polydopamine (PDA) and pheomelanin-like PDA/cysteine codeposited coatings on the hair surface. The color and morphology of the resulting hair were characterized in detail by several spectroscopy methods and the possible mechanism for the multicoloring effects and structural differences of the melanin-mimetic coating was proposed. Our strategy eliminates the use of toxic dye precursors or organic solvents, and the favorable safety of the PDA-based formulations is demonstrated. The fabricated dyes can be applied to hair simply by combing, resulting in uniform multi-coloring effects within a short time. Furthermore, the melanin-mimetic hair dyes have excellent durability and ultraviolet protection performance. This work provides a facile and versatile methodology to develop the next generation of safe, sustainable and multicolor hair dyes and pave new avenues for advancing the field of surface coloration, nanoreactors, nanogenerators, energy storage materials and biomimetic sensing devices.

Received 16th September 2019 Accepted 13th October 2019

DOI: 10.1039/c9ra07466j

rsc.li/rsc-advances and graphene-based sheets ${ }^{7}$ as colorants for hair coloring. However, these methods still have some drawbacks, such as poor long-term durability of coloration, tedious synthesis and extraction processes, potential toxicity, ${ }^{8}$ and limited hair color variety. Thus, the development of new dyeing ingredients and surface coloration approaches for hair dyeing is highly significant.

Nature provides plenty of colors and shades for human hair ranging from light blond, brown, red to black, which are determined by the quantity and ratio of two types of melanin pigments exist in hair, i.e. the black-brown eumelanins and the reddish-yellow pheomelanins. ${ }^{2}$ Unlike the black hair exhibiting high levels of eumelanins formed by the oxidative polymerization of dopaquinone, the red hair has a large quantity of pheomelanins biosynthesized from 5-S-cysteinyldopa, the oxidative coupling product of dopaquinone with cysteine. ${ }^{9}$ On the other hand, polydopamine (PDA) has attracted extensive interest in many research fields ${ }^{\mathbf{1 0 , 1 1}}$ because of its many fascinating properties such as versatile adhesion capability to almost any substrate, excellent biocompatibility, chemical reactivity, etc. $^{12-15}$ More remarkably, as an analogue of natural eumelanin, PDA preserves similar structures and physicochemical properties as its natural resources, ${ }^{\mathbf{1 1}, 16}$ thus possessing enormous potential as a eumelanin-like pigment in the research field of surface coloration. However, up to now, the application focused on the PDA-based surface coloration is rare. One crucial issue is
Institute of Engineering Thermophysics, School of Energy and Power Engine Chongqing University, Chongqing 400030, China.E-mail: lijun@cqu.edu.cn ${ }^{b}$ School of Pharmacy, Southwest Medical University, Luzhou, Sichuan, 646000, China. E-mail: fuqifeng1990@163.com

'Analytical and Testing Center, Chongqing University, Chongqing, 401331, China

${ }^{d}$ School of Pharmaceutical Sciences, Chongqing University, Chongqing, 401331, China $\dagger$ Electronic supplementary information (ESI) available. See DOI: 10.1039/c9ra07466j

\$ These authors contributed equally. 
the slow formation rate of PDA, which usually takes more than ten hours or even a few days. ${ }^{12}$ Another issue is that PDA coatings typically present a single color of brown-black, which greatly limits the potential of PDA in colorants. Recently, the methods using PDA to generate deep black coloring effect on human hair in the presence of ferrous ions or copper ions were proposed. ${ }^{17,18}$ Regrettably, the surface contamination of the transition metal oxidants on the surface of hair is unavoidable, and the potential risk of metal ions exposure for human cannot be ignored. Besides, none of them can attain diverse coloring effects but black, which is difficult to meet the diversified demands for hair dyeing colors. Therefore, it remains a huge challenge to develop highly practical multicolor PDA coating on hair surface for realizing diverse hair coloring effects.

Herein, inspired by the melanin-based coloring mechanism of human hair and excellent melanin-like properties of PDA, we report a novel strategy for fabricating melanin-mimetic multicolor and low-toxic PDA coating on the hair surface, through sodium periodate (SP) induced in situ rapid deposition of eumelanin-like PDA and pheomelanin-like PDA/cysteine codeposited coatings (Fig. 1). In our cases, the existing crucial issues affecting the practicability of PDA in hair surface coloration are addressed through a comprehensive approach in which: (1) the rapid deposition of PDA coatings on hair surface is realized using SP as a trigger, (2) the significant influences of periodate oxidation conditions on the color and shade of PDA coatings on hair surface are revealed, (3) a novel pheomelanininspired PDA/cysteine co-deposition strategy is developed to obtain red coloring effects. The color and morphology of the multicolor PDA coatings were detailedly investigated by scanning electron microscope (SEM) and several other spectroscopy methods, which verified the significant impacts of periodate oxidation conditions on the chemical components and colors of PDA coatings. A possible mechanism underlying the multicoloring effects and structure differences of PDA coating was proposed, which is related to the change of coating thickness and the conversion of hydroxyl groups into carbonyl/carboxyl groups in PDA coatings. The PDA-based formulations consist of four main components, including dopamine (DA) and cysteine as coating precursors, SP as the trigger for the rapid deposition of PDA, ${ }^{19,20}$ the acetate buffer as the solvent and Pluronic F127 (PF127) as the gelatinizer for forming hydrogel. The resulting PDA-based colorants can be applied to hair simply by combing, leading to uniform multicolor PDA coatings on hair surface within a short time. In addition, the melaninmimetic coating has excellent UV protection performance and can resist repetitive washes with shampoo, which is comparable with commercial permanent hair dyes. Their acute dermal toxicity to mice skin is also evaluated and no evidence of toxicity and irritation can be found.

\section{Experimental}

\section{Materials}

Dopamine hydrochloride, L-cysteine, sodium periodate, sodium acetate trihydrate, acetic acid and polyvinylpyrrolidone (PVP, MW $10000 \mathrm{Da}$ ) were all purchased from Adamas Reagent Co., Ltd (Shanghai, China). Tris (hydroxymethyl) aminomethane, sodium carboxymethyl cellulose (CMC, 300-800 mPa s), chitosan (CTS, 200-400 mPa s), polyethylene glycol (PEG, MW $10000 \mathrm{Da}$ ) and xanthan gum (XTG, USP grade) were obtained from Aladdin Reagent Co., Ltd (Shanghai, China). Carbomer 940 (C940) was purchased from Macklin Biochemical Co. Ltd. (Shanghai, China). Pluronic F127 was bought from Sigma-Aldrich (St. Louis, MO, USA). Human hair samples (\#60 platinum blonde) was purchased from Ruolani Hair Products Co., Ltd (Lishui, China). The shampoo (Head \& shoulders) was obtained from a supermarket in Luzhou, China. The ultrapure water used in this study was purified with an AWL-1002-H (Aquapro International Company LLC., USA) ultra-pure water system.

\section{Hair dyeing with PDA solutions}

Initial PDA-based hair coloring was conducted by using the aqueous solutions of DA. Briefly, different concentration of DA

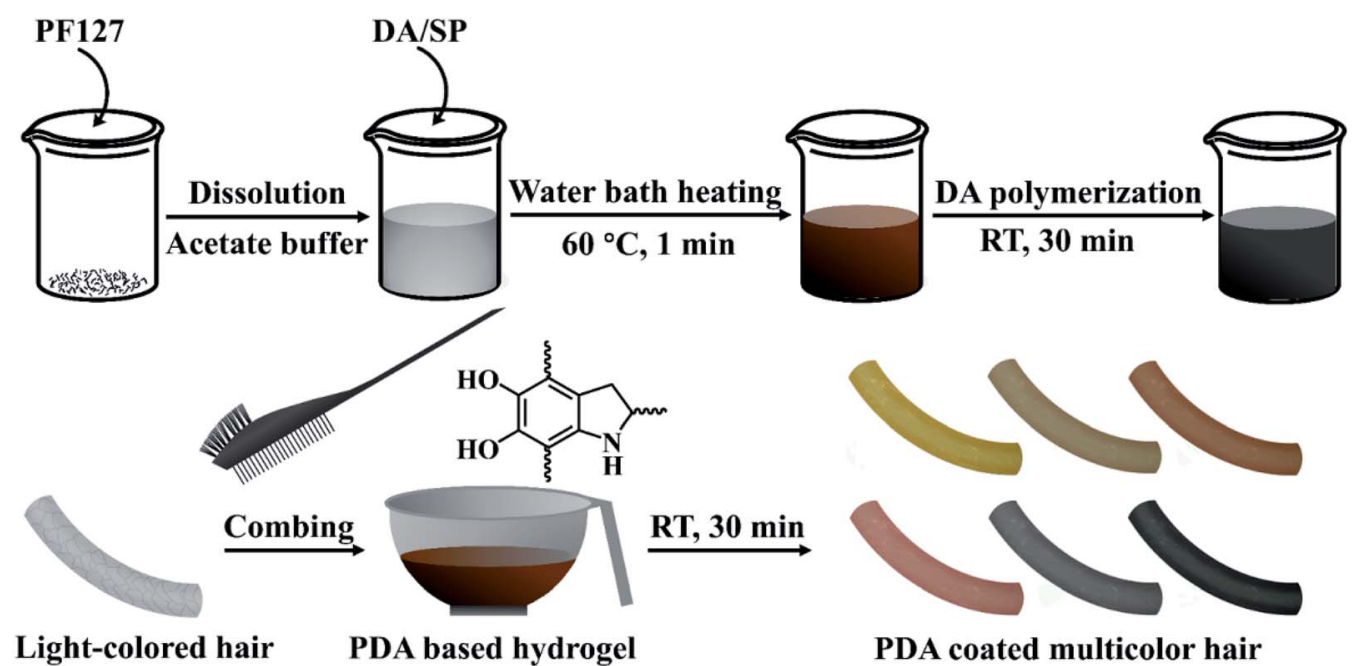

Fig. 1 Schematic of the preparation and coloring procedures of multicolor PDA coating-based hair dyes. 
was firstly dissolved into $20 \mathrm{~mL}$ of sodium acetate buffer (50 $\mathrm{mM}, \mathrm{pH}=5$ ) and then SP with the desired concentration was added into the DA solution. After that, a bunch of hair (about $1 \mathrm{~g}$ ) was soaked in the DA/SP solution immediately. The coloring processes were performed at room temperature for a desired time (typically $30 \mathrm{~min}$ ). The obtained PDA-coated hair samples were washed with running water and dried with a domestic hair dryer.

\section{Hair dyeing with PDA hydrogels}

In order to improve the operability of PDA-derived hair-dyeing in practice, the PDA based hydrogel formulation was prepared by mixing DA with PF127 in the presence of SP. Briefly, PF127 (25 wt\%) was first dissolved in $20 \mathrm{~mL}$ of sodium acetate buffer $(50 \mathrm{mM}, \mathrm{pH}=5)$ at $4{ }^{\circ} \mathrm{C}$ in a refrigerator before adding DA and SP as described above. The resulting mixed solution was heated in a water bath at $60^{\circ} \mathrm{C}$ and the PDA based hydrogel was rapidly formed because of the reverse thermo-responsive phase transition property of PF127. Then, the as-prepared dye hydrogel was combed onto the hair uniformly and left to stand at room temperature for $30 \mathrm{~min}$. Subsequently, the dyed hair samples were rinsed well and dried with a hair dryer.

\section{Hair dyeing with PDA/cysteine hybrid hydrogels}

To further enrich the color spectrum of the PDA based dyes, the PDA/cysteine hybrid hydrogels were prepared by mixing L-cysteine with PDA hydrogels. Briefly, different concentration of L-cysteine and DA were firstly co-dissolved into the aforementioned PF127 (25 wt\%) solution before adding SP with the desired concentration. The resulting mixed solution was heated in a water bath at $60{ }^{\circ} \mathrm{C}$ and the PDA/L-cysteine hybrid hydrogel was rapidly formed. Next, the as-prepared dye hydrogels were combed onto the hair uniformly and left to stand at room temperature for $30 \mathrm{~min}$. Subsequently, the dyed hair samples were processed by washing and drying.

\section{Durability measurement}

The PDA-dyed hair samples were completely soaked in $40 \mathrm{~mL}$ of 5 vol\% shampoo solutions and encapsulated into $50 \mathrm{~mL}$ centrifuge tubes (JET). The tubes were then vigorously shaken for $5 \mathrm{~min}$ with a vortex mixer. After shampooing, the hair samples were further rinsed with running water and dried by a hair dryer. A total of 30 shampooing cycles were conducted for each sample.

\section{Acute dermal toxicity experiments}

All animal procedures of the acute dermal toxicity experiments were in accordance with the Guidelines for Care and Use of Laboratory Animals of Southwest Medical University and the experiments were approved by the Animal Ethics Committee of Southwest Medical University Animal Center (permit no. 20160126). Healthy male SD mice were purchased from Dashuo Biotechnology Co. Ltd (Chengdu, China). Hair from the dorsal side of mice were removed by using an electric clipper. A $25 \mathrm{~mm}$ $\times 25 \mathrm{~mm}$ section of absorbent gauze patch was saturated with the freshly prepared PDA-based dyes $\left(2000 \mathrm{mg} \mathrm{kg}^{-1}\right)$ and then was applied to the depilated area of the animals for $24 \mathrm{~h}$. The control animals received the same quantity of sodium acetate buffer in a similar manner. At the end of the contact time, the patches were removed. The symptom, body weight and mortality were recorded carefully every day for 14 days.

\section{Characterization}

The color of dyed hair was measured using a precision colorimeter (NR110, 3NH Technology Co., Ltd, Shenzhen, China). For each hair sample, the color coordinate values, including $L^{*}$, $a^{*}$ and $b^{*}$, were measured ten times to calculate the mean value. Specifically, $L^{*}$ represents lightness value (lower $L^{*}$ values are darker), $a^{*}$ indicates the chromaticity from red (positive values) to green (negative values), $b^{*}$ represents the chromaticity from blue (negative values) to yellow (positive values). UV-visible absorption spectra of PDA solutions and PDA coated hair were obtained using a UV-visible spectrophotometer (UV-3600, Shimadzu, Japan). The morphology observation of hair was performed by using SEM (S4800, Hitachi, Japan). The elementary chemical composition of PDA coated hair was confirmed by XPS spectrometer (ESCALAB 250Xi, Thermo Electron, USA). The surface chemical components of the PDA coatings deposited on the surface of quartz plate, which preparation processes were same as that deposited on the hair surface, were investigated by ATR-FTIR spectroscopy (Nicolet iS50, Thermo Scientific Inc., USA).

\section{Results and discussion}

\section{PDA-based eumelanin-like multicolor hair dye}

Platinum blonde hair samples were utilized for evaluating the coloring performance of the PDA coatings on hair surface. The coloring process was conducted by using SP as an oxidant under a slightly acidic condition ( $\mathrm{pH}$ 5.0), which could avoid the opening of the cuticles and reduce the permanent damage to hair. ${ }^{7}$ Fig. 2c shows the UV-visible absorption spectra of the DA/ SP coating solutions obtained at different times. Upon addition of SP, the absorption peak at around $480 \mathrm{~nm}$ was observed within a few minutes, which might be caused by the intramolecular cyclization of dopamine quinone to dopaminochrome. ${ }^{21,22}$ With the extension of reaction time, its intensity increased rapidly and the color of the DA/SP solution darkened dramatically to deep black within 30 min (Fig. 2a). In contrast, the color and absorbance of the alkaline DA solution (Tris-HCl buffer, $\mathrm{pH} 8.5,50 \mathrm{mM}$ ) changed little in the same time (Fig. 2a and b). Thus, the polymerization rate of DA was greatly improved via SP-induced oxidation. Next, the color change of PDA coated hair obtained at different reaction times was further investigated. As time progressed, the color of the PDA coated hair rapidly changed from platinum blonde to dark black. After $30 \mathrm{~min}$ of coating, the appearance of PDA coated hair was close to that of a commercial black hair dye (Fig. 3a). Fig. 2f exhibits the typical SEM images of PDA coated hair obtained at different times. As shown, with the coating time increased, the uniform coating deposited on the hair surface 


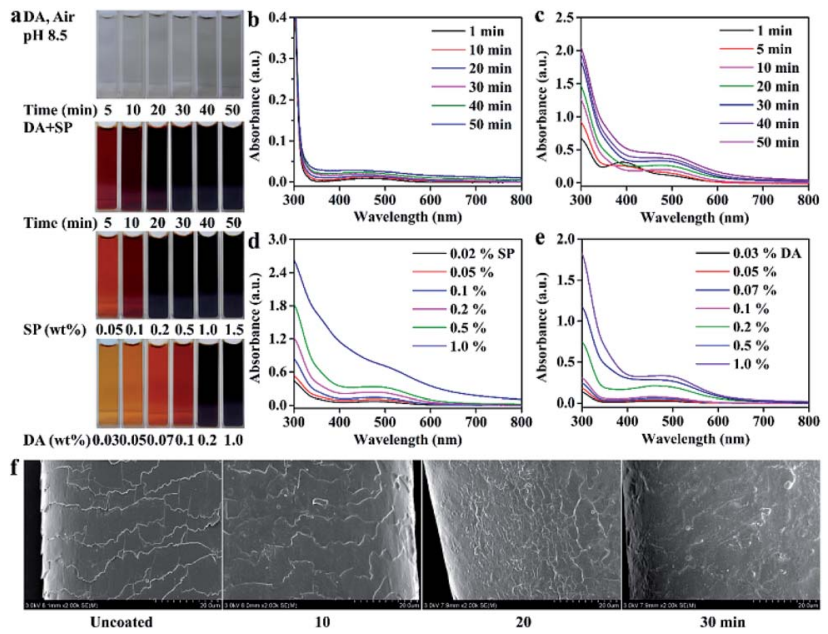

Fig. 2 Effect of reaction times, concentrations of SP and DA on (a) color and $(b-e)$ UV-visible absorption properties of DA/SP solutions (DA solutions were diluted 60-fold before the spectral analysis). (f) SEM images of uncoated and PDA coated hair obtained at different reaction times.

was increasingly thickened and the cuticle scales gradually became unapparent within $30 \mathrm{~min}$, which verified the in situ rapid deposition of PDA coating on the hair surface. The difference in the color of PDA coating on hair surface was also quantified by the determination of lightness (Fig. 3b), which showed that the lightness of PDA coated hair decreased continuously with the increase of coating time, reaching a level similar to that of control in $30 \mathrm{~min}$. Thus, the presented PDA deposition-based dyeing process is faster than the aromatic amine-based coloring treatments depending on the molecule diffusion and can take hours to complete. Moreover, unlike the commercial permanent hair dyes changing the color of hair by the deep penetration of aromatic amine-based colorants inside the hair, the PDA-based coatings adhered on the surface of hair are less likely to cause permanent hair damage.

The color and UV-visible spectra of the PDA solutions after 30 min of oxidation with different amounts of SP were recorded (Fig. 2a and d). With the increase of SP concentration, the color of the PDA solutions sharply deepened from brown to dark black and the corresponding absorbance at around $480 \mathrm{~nm}$ increased and shifted toward long wavelength markedly. Thus, the color and formation rate of PDA were found to be significantly affected by SP concentration, consistent with previous studies. ${ }^{19,20}$ Fig. 3d exhibited the color change of PDA coatings on hair surface triggered by different amounts of SP. The color of the hair samples changed inconspicuously after soaking in alkaline DA solution for $30 \mathrm{~min}$, in the absence of SP. In contrast, gradient shades of black could be easily obtained by the introduction of increased amounts of SP as the oxidant under slightly acidic conditions, corresponding to the gradually decreased lightness (Fig. 3f). A small amount of SP (0.5 wt\%) could enormously enhance the hair coloring effect of PDA, approaching that of a commercial black hair dye. Therefore, the concentration of SP has a crucial regulating effect on the polymerization and rapid coloring of PDA on hair.
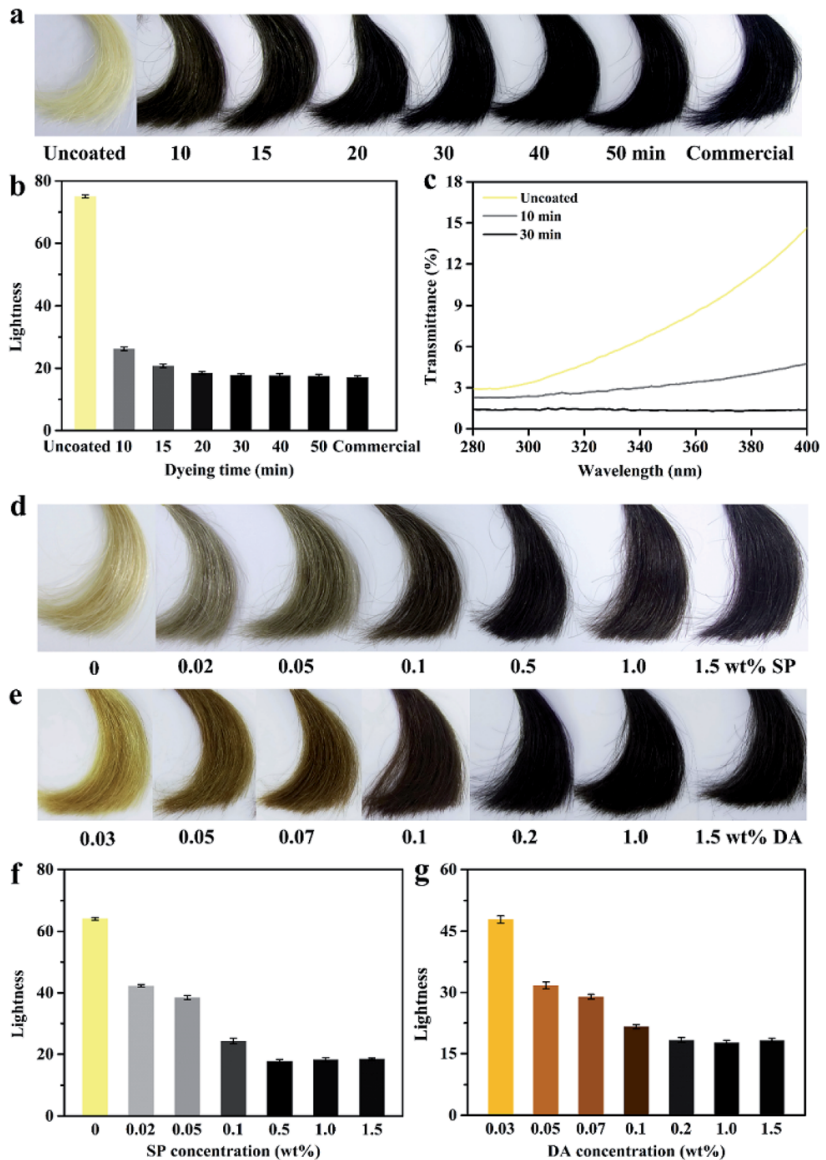

Fig. 3 Photographical images (a) and lightness (b) of uncoated and PDA coated hair obtained at different reaction times, in comparison with another hair sample dyed with a commercial permanent black hair dye. UV transmittance (c) of uncoated and PDA coated hair obtained at different reaction times. Photos of PDA coated hair after 30 min deposition with different concentrations of (d) SP and (e) DA. The light-ness of PDA coated hair obtained using different concentrations of (f) SP and (g) DA, determined with a precision colorimeter.

Fig. 2e exhibits the UV-visible spectra of DA solutions after 30 min of oxidation using different concentrations of DA. With the increase of DA concentration, the characteristic absorption peaks corresponding to the intramolecular cyclization of dopamine quinone to dopamine-chrome gradually red-shifted from $450 \mathrm{~nm}$ to $480 \mathrm{~nm}$ and the corresponding peak intensity increased continuously. Meanwhile, the color of the DA/SP solution gradually changed from light brown, brown to dark black (Fig. 2a). Motivated by the higher polymerization rate of DA and remarkable color change of PDA with the increase of DA amount, we further investigated the effect of DA concentration on the color of PDA coatings on hair surface. As shown in Fig. 3e, besides the dark black color obtain by using $1.0 \mathrm{wt} \% \mathrm{DA}$, PDA-coated hair with a wider variety of colors, including light brown, brown and dark brown could be easily obtained by varying the concentration of DA, corresponding to the continuously decreased lightness (Fig. 3g). The black to brown multicolor appearances of PDA coatings on hair surface are consistent with the color spectrum of naturally occurring 
eumelanins in human hair and contribute to meeting the growing demands for multicolor hair treatment.

The structure differences of as-prepared PDA coating with different periodate oxidation conditions were further investigated by ATR-FTIR and XPS measurements. ATR-FTIR spectra in Fig. 4 show a series of new absorption peaks derived from PDA for all PDA coatings formed with different amounts of SP and DA. The absorption band from $3100 \mathrm{~cm}^{-1}$ to $3700 \mathrm{~cm}^{-1}$ and the absorption peaks at 2920, 2850, 1610, 1500 and $1445 \mathrm{~cm}^{-1}$ can be assigned to the vibrations of catechol $-\mathrm{OH}, \mathrm{N}-\mathrm{H}$, methylene groups and benzene skeleton from PDA, respectively. In addition, the spectra of the PDA samples obtained using higher SP concentration $(0.5 \mathrm{wt} \%)$ show a new absorption peak around $1720 \mathrm{~cm}^{-1}$, assigned to carbonyl/carboxyl groups. ${ }^{19}$

XPS results (Fig. 5a and b) also indicate that the percentage of $\mathrm{C}=\mathrm{O}$ bond in PDA coating greatly rose from $1.7 \%$ to $11.8 \%$ with the concentration of SP increase from $0.2 \mathrm{wt} \%$ to $1.0 \mathrm{wt} \%$, denoting that higher concentrations of SP can lead to higher degree of oxidation and higher proportion of carbonyl/carboxyl groups in PDA. Additionally, the concentration of DA also can significantly affect the abundance of oxygenated carbon in PDA (Fig. 5c and d). PDA coating formed with $0.5 \mathrm{wt} \%$ DA has a higher ratio of $\mathrm{C}-\mathrm{O}$ and $\mathrm{C}=\mathrm{O}$ bonds than that obtained with $0.1 \mathrm{wt} \%$ DA. The significant impacts of periodate oxidation conditions on the abundance of carbonyl and carboxyl groups in PDA coatings are in accord with the two-electron oxidant properties of periodate, i.e., high concentrations of periodate can induce the cleavage of oxidative $o$-quinone, resulting in the partial loss of aromatic rings and higher content of carbonyl/ carboxyl groups in PDA. ${ }^{19,20}$ which may lead to the change in the visible light absorption properties of PDA and the intriguing multi-coloring hair dyeing effects.

Based on the characterization results of SEM, ATR-FTIR and XPS measurements, the underlying mechanisms of multicolor appearances of PDA coatings on hair surface are probably associated with the coating thickness and the conversion of hydroxyl groups into carbonyl/carboxyl groups in PDA coatings. Specifically, the thickness of PDA coating rapidly increased with the increase of coloring time, leading to the enhanced visible
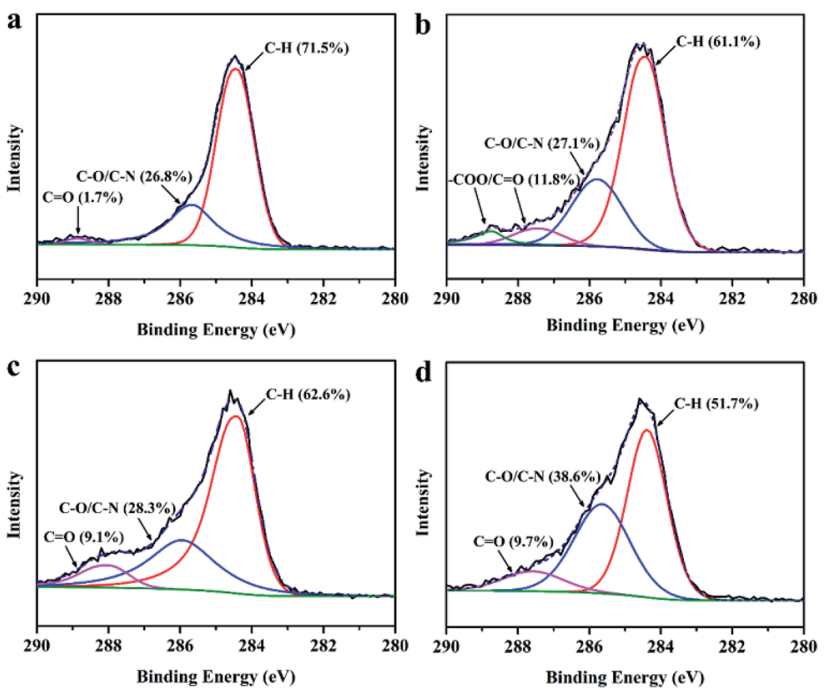

Fig. 5 High-resolution $C$ 1s XPS spectra of PDA coatings on hair surface synthesized with (a) 0.2 wt $\%$ SP, (b) 1.0 wt\% SP (DA concentration was $1.0 \mathrm{wt} \%$ ); (c) $0.1 \mathrm{wt} \% \mathrm{DA}$ and (d) $0.5 \mathrm{wt} \%$ DA (SP concentration was $0.5 \mathrm{wt} \%)$ for $30 \mathrm{~min}$.

light absorption and deepened shades. On the other hand, during the periodate oxidation reaction, different amounts of phenolic hydroxyl groups would convert to carbonyl and carboxyl chromophores along with the change of DA and SP concentrations, which may cause the observed change in visible light absorption properties of PDA and the intriguing multicoloring effects.

\section{Eumelanin-like hydrogel hair dye}

To improve the practical operability of the multicolor PDA coating-based hair coloring strategy, we further developed the PDA-based hydrogel formulation by mixing several common thickeners, including chitosan, xanthan gum, polyethylene glycol, polyvinylpyrrolidone, carbomer 940 and sodium carboxymethyl cellulose, into the DA/SP solutions. However, as shown in Fig. S1a and $c, \uparrow$ although the increases in the viscosity
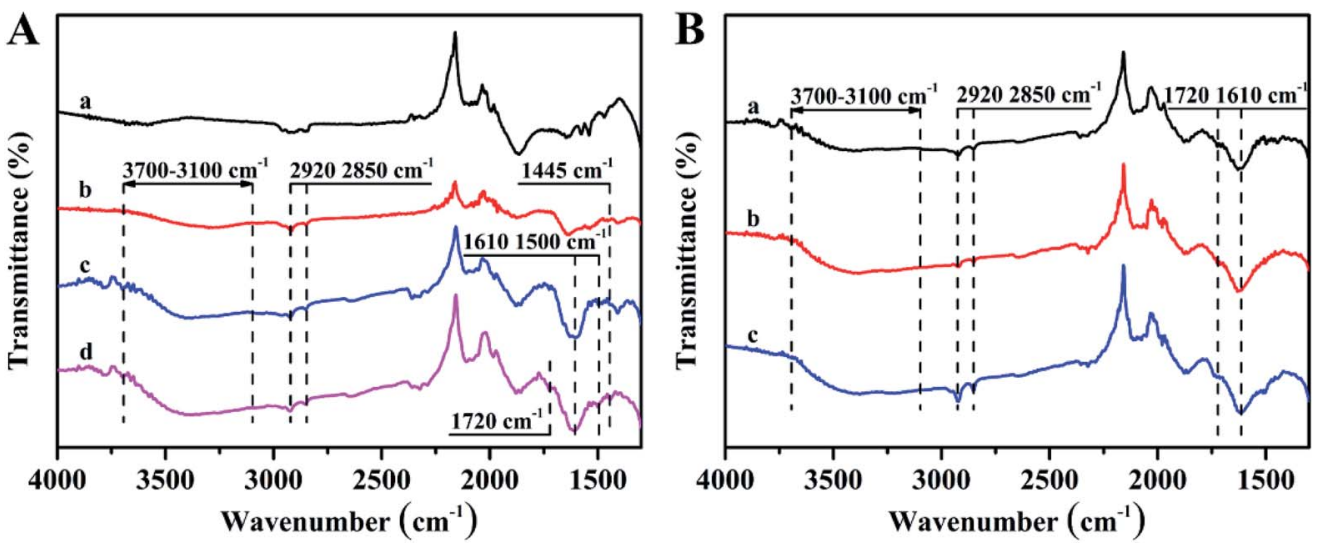

Fig. 4 (A) ATR-FTIR spectra of uncoated quartz plate (curve a), PDA coatings oxidized by 0.1 wt\% (curve b), 0.2 wt $\%$ (curve c) and 0.5 wt\% (curve d) SP (DA concentration was 1.0 wt\%) for 30 min; (B) ATR-FTIR spectra of PDA coatings synthesized with 0.1 wt\% (curve a), 0.2 wt $\%$ (curve b) and $0.5 \mathrm{wt} \%$ (curve c) DA (SP concentration was $0.5 \mathrm{wt} \%$ ) for $30 \mathrm{~min}$. 
of PDA solutions could be achieved, different degrees of degradation in the coloring effects were observed after being mixed with these thickeners, which might be due to the inhibition of the polymerization and deposition of DA. In contrast, unchanged coloring effects of PDA-coated hair were observed after introduction of PF127, a non-toxic lock copolymer. ${ }^{23}$ With the increase of PF127 amount, the color and lightness of the PDA coated hair remained virtually unchanged after $30 \mathrm{~min}$ of coating (Fig. S1b and d $\dagger$ ), indicating that PF127 had no significant impact on the polymerization and deposition of DA. When 25 wt\% PF127 was mixed with DA and SP, the PDA/PF127 hydrogel could be easily formed at room temperature (Fig. 6a) because of the inverse thermo-gelling property of PF127. ${ }^{23}$ The PDA-based hydrogel formulation could be directly applied to hair dyeing simply by combing (Fig. 6b) because of the strong adhesion of the PDA/PF127 hydrogel to hair. Consequently, a variety of coloring effects, similar to those obtained with PDA solutions, can be easily achieved by adjusting the preparation parameters of the PDA/PF127 hydrogels (Fig. S2 $\dagger$ ).

\section{Color fastness of PDA coated hair}

The color fastness of the PDA coated hair samples was evaluated by exhaustive washing tests with shampoo. As shown in Fig. 7b-d, the typical PDA coated hair samples with different colors remained their intrinsic colors after thirty shampoo washes. Correspondingly, their lightness also had no significant change during the shampoo washing processes (Fig. 7e), which suggest the detachment of PDA coatings hardly occurred. The excellent color fastness of PDA-coated hair can be attributed to the strong interaction between PDA and hair surface. More specifically, the PDA-based coatings can strongly adhere on virtually all types of surfaces due to its durable stability and robust adhesion. ${ }^{\mathbf{1 1}}$ Furthermore, the PDA-based coatings have strong covalent reactivity with the amine and thiol-containing keratin molecules on the hair surface, which also contribute to the firm immobilization of PDA coating on hair. ${ }^{\mathbf{1 1 , 1 5}}$ As for the commercial permanent hair dyes, they can maintain their coloring effects for a long time

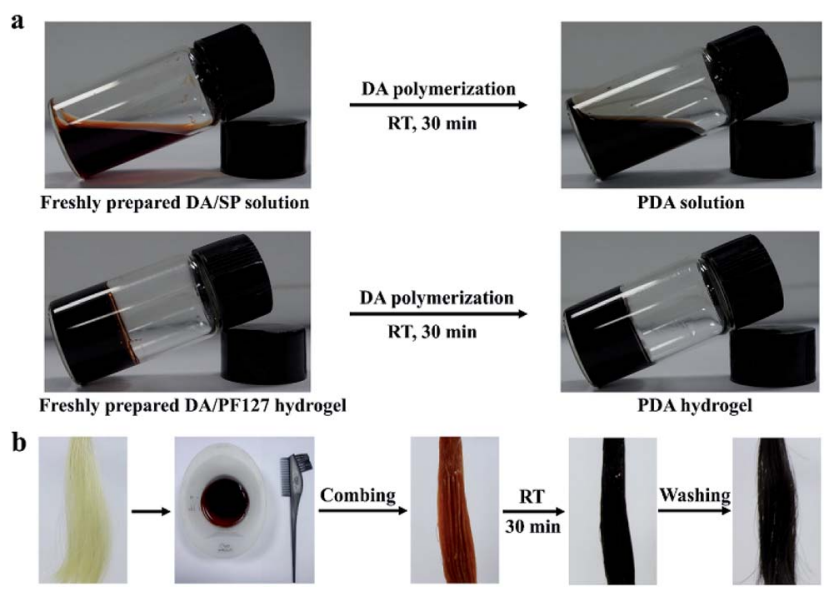

Fig. 6 (a) The thermo-responsive phase transition property of PDA/ PF127 hydrogel; (b) the hair dyeing process of PDA/PF127 hydrogelbased hair dyes.
(Fig. 7a) owing to the irreversible changes in hair composition induced by the coloring reactions. However, their coloring operations have to be repeated almost every month because of hair growth. Thus, it can be confirmed that the PDA hydrogel-based hair colorant formulation possess excellent durability, which is comparable with the permanent coloring effect of commercial hair dyes.

\section{UV protection performance}

In addition to the function of multicolored and permanent coloration, the PDA-based hair colorant formulation can potentially render more functionalities to hair such as UV protection because of the strong UV absorbance property of PDA. The gray hair with reduced amount of melanins, the natural photo-protective substance, are more susceptible to the damage caused by UV radiation, resulting in higher rate of disulfide bonds break and lower mechanical strength. ${ }^{24}$ Thus, it is essential to provide a barrier against UV radiation for the gray hair. The possible UV protection property of PDA-based hair coating was further studied by the determination of UV transmittance. As shown in Fig. 3c, in the range of $280-400 \mathrm{~nm}$, the UV transmittance of uncoated light-colored hair rapidly increased with the increase of wavelength, reaching a maximum of over $15 \%$ transmittance. In contrast, the PDA coated hair samples exhibited lower UV transmittance. Especially, the transmittance of the PDA coated dark black hair was close to zero, which was sufficient for UV protection purposes. The excellent UV protection property of PDA-based hair colorant formulation can be ascribed to their broadband absorption property similar to naturally occurring eumelanin. ${ }^{\mathbf{1 1 , 2 5}}$

\section{PDA/cysteine-based pheomelanin-like red hair dye}

Although several coloring effects have been achieved by tuning the periodate oxidation conditions, the diversity of coating colors was still limited. In contrast, the hair and wool of mammals have a much wider variety of colors, which are determined by the quantity and ratio of black-brown eumelanins and reddish-yellow pheomelanins. ${ }^{2}$ Inspired by the formation mechanism of natural pheomelanins, we utilized PDA/cysteine co-deposition strategy to further enrich the color spectrum of the PDA-based hair coatings. More specifically, different amounts of cysteine were co-dissolved with DA in the presence of SP and PF127 to obtain the cysteine-doped PDA/ PF127 hydrogels. During their subsequent coating processes, the SP-induced polymerization of DA and its copolymerization with cysteine could be proceeded simultaneously, ${ }^{26}$ resulting in the co-deposition of PDA and the pheomelanin analogues, i.e., DA/cysteine copolymers onto hair surfaces. Accordingly, the hair samples with different shades of red were easily obtained by varying the doping amounts of cysteine (Fig. 8a), corresponding to the color coordinate values (Fig. 8c). With the increased amount of cysteine, the color of the PDA/cysteine coated hair shifted toward red, which could be attributed to the continuous increase of the proportion of the pheomelanin analogues deposited on hair surface. Moreover, the obtained PDA/cysteine co-deposited coating had good durability, 

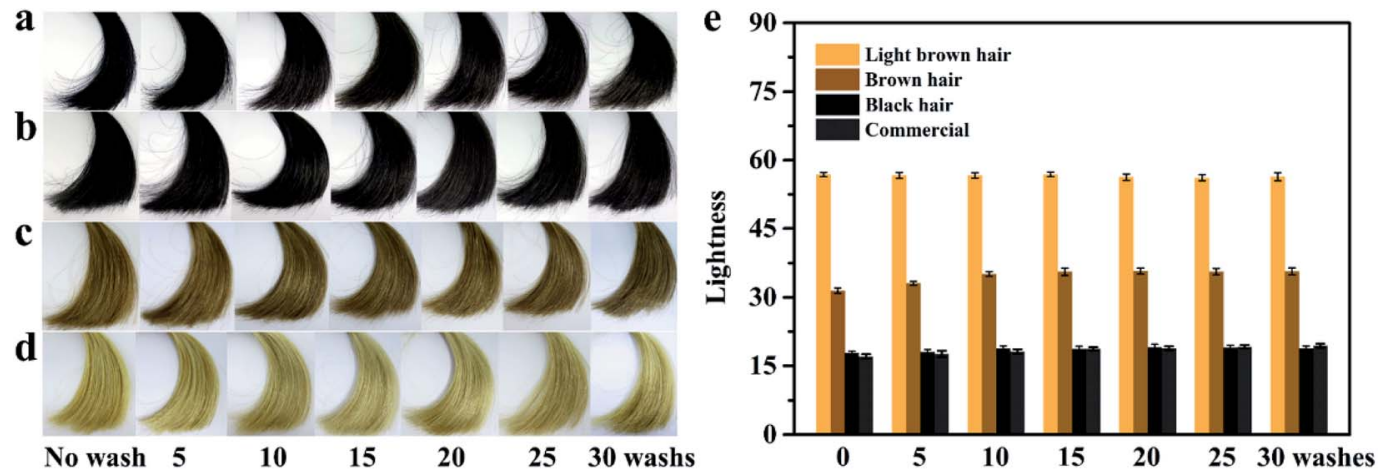

Fig. 7 Photographical images of hair samples dyed with (a) commercial permanent black dye, (b) dark black-colored, (c) brown-colored and (d) light brown-colored PDA/PF127 based hair dyes before and after repeated shampoo washing. (e) The corresponding changes in lightness of each hair samples before and after exhaustive shampoo washing

confirmed by the exhaustive washing tests (Fig. $8 \mathrm{~b}$ and d). Therefore, the pheomelanin-inspired co-deposition strategy not only provides a novel and effective route for designing red color melanin-mimetic hair colorants, but also contribute to deepen the understanding of the hair coloring mechanisms of naturally occurring pheomelanins. The existence of the cross-linking reaction between DA and cysteine was verified by UV-visible absorption spectra. As shown in Fig. S3.† PDA/cysteine solution shows a relatively strong absorption around $350 \mathrm{~nm}$, which indicating the formation of $\mathrm{C}=\mathrm{C}-\mathrm{C}=\mathrm{S}$ and $\mathrm{C}=\mathrm{C}-\mathrm{C}=\mathrm{N} \cdot{ }^{27}$ Besides, a markedly blue shift of the absorption peak from $480 \mathrm{~nm}$ to $440 \mathrm{~nm}$ was also observed, which may be related to the decrease of carbonyl/carboxyl group content. In other words, the polymerization pathway of PDA is partially converted to the formation of cysteinyl conjugate because of the doping of cysteine. With increasing mass ratio of cysteine, the covalent interaction of DA with cysteine strengthened gradually and the polymerization process of DA was gradually inhibited in the presence of excessive cysteine, corresponding to the gradually decreased of peak intensity. Thus, the measurements of UVvisible absorption spectra further confirmed that the red coloring effects were caused by the formation of pheomelanin-

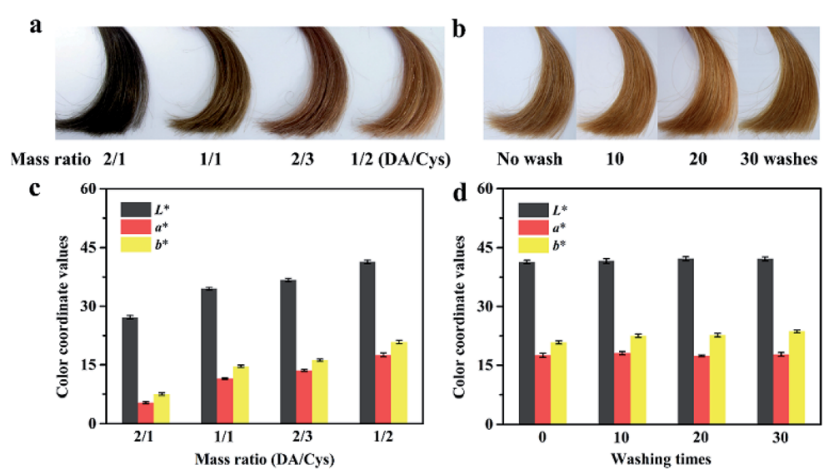

Fig. 8 (a) Photographical images and (c) color coordinate values of PDA/cysteine co-deposited hair obtained using different doping amounts of cysteine (DA concentration was $1.0 \mathrm{wt} \%$ ). (b) Photographs and (d) color coordinate values of red-colored hair obtained through PDA/cysteine $1: 2$ co-deposition before and after repeated shampoo washing. like PDA/cysteine copolymer, which arised from the crosslinking reaction between DA and cysteine.

\section{In vivo toxicity assessment}

The presented PDA-based hair colorant formulation are anticipated to be safer than commonly used commercial permanent hair dyes for the following reasons: (i) the PDA-based hydrogel formulations contain no organic solvents or toxic aromatic aminebased colorants; (ii) as an analogue of naturally occurring eumelanin, PDA has been proved to possess good biocompatibility and negligible in vitro and in vivo toxicity; ${ }^{28-30}$ (iii) the highly hydrophilic precursor DA and the synthetic insoluble PDA with high molecular weight are unlikely to penetrate the skin barrier. ${ }^{31,32}$ To verify the prediction, we assessed the acute dermal toxicity of the presented PDA-based hair colorant formulation using SD mice. The whole mice whose skins were subjected to the PDA solutions or PDA/PF127 hydrogels at the dose of $2000 \mathrm{mg} \mathrm{kg}^{-1}$ remained healthy within 14 days. The weight of the PDA/PF127 treated groups steadily increased in the manner similar to that of the control group treated by sodium acetate buffer and no evidence of irritation could be observed throughout the test period (Fig. S4 and Table S1 $\dagger$ ). Thus, the PDA/PF127 hydrogel-based colorant formulation showed a high median lethal dose value greater than $2000 \mathrm{mg} \mathrm{kg}^{-1}$, indicating its negligible acute dermal toxicity.

\section{Conclusions}

In summary, we have developed a facile and versatile surface coloration method for fabricating melanin-mimetic multicolor and low-toxic hair dyes through SP-triggered rapid deposition of eumelanin-like PDA and pheomelanin-like PDA/cysteine codeposited coatings on the hair surface. The PDA-based formulations contain no toxic aromatic amine-based colorants or organic solvents, and their safety was confirmed by the acute dermal toxicity tests. The melanin-mimetic hair dyes have excellent practicability, durability and UV protection performance. More intriguingly, in addition to gradient shades of black, PDA coated hair with more diversity of colors, which are consistent with the color spectrum of human natural hair, could be easily obtained by tailoring the formation conditions of PDA 
and the ratio of eumelanin and pheomelanin analogues. The possible mechanism underlying the multi-coloring effects and structure differences of PDA-based hair coating was proposed. This work provides a novel biomimetic strategy to develop the next generation of sustainable, safe, and multicolor hair dyes, which may also pave novel pathways for advancing the field of surface coloration. For instance, this strategy can be utilized for the coloration of various textile materials resistant to coloration. In addition to cosmetic and coloration, PDA coating can also render many new potential functionalities to hair because of its remarkable physicochemical versatility. ${ }^{\mathbf{1 0 , 1 1 , 3 3}}$ Therefore, this PDA coated hair can be easily re-functionalized and extended to other related areas such as nanoreactors, ${ }^{6,34}$ nanogenerators, ${ }^{35}$ energy storage materials ${ }^{36}$ and biomimetic sensing devices..$^{37,38}$

\section{Conflicts of interest}

There are no conflicts to declare.

\section{Acknowledgements}

This work was supported by the National Natural Science Foundation of China (Grant No. 21804113 and 51622602), the Science and Technology Transformation Project of Southwest Medical University (Grant No. 2018001), the Talent Introduction Project of Southwest Medical University (Grant No. 090300040005) and the Research Fund of Southwest Medical University (Grant No. 2017-ZRQN-030).

\section{References}

1 A. B. Lerner, Am. Med. J., 1971, 51, 141-147.

2 O. J. X. Morel and R. M. Christie, Chem. Rev., 2011, 111, 2537-2561.

3 M. Harling, A. Schablon, G. Schedlbauer, M. Dulon and A. Nienhaus, Occup. Environ. Med., 2010, 67, 351-358.

4 C. Boga, C. Delpivo, B. Ballarin, M. Morigi, S. Galli, G. Micheletti and S. Tozzi, Dyes Pigm., 2013, 97, 9-18.

5 P. M. Rose, V. Cantrill, M. Benohoud, A. Tidder, C. M. Rayner and R. S. Blackburn, J. Agric. Food Chem., 2018, 66, 6790-6798.

6 S. D. Haveli, P. Walter, G. Patriarche, J. Ayache, J. Castaing, E. Van Elslande, G. Tsoucaris, P.-A. Wang and H. B. Kagan, Nano Lett., 2012, 12, 6212-6217.

7 C. Luo, L. Zhou, K. Chiou and J. Huang, Chem, 2018, 4, 784794.

8 K.-H. Liao, Y.-S. Lin, C. W. Macosko and C. L. Haynes, ACS Appl. Mater. Interfaces, 2011, 3, 2607-2615.

9 M. Ambrico, N. F. D. Vecchia, P. F. Ambrico, A. Cardone, S. R. Cicco, T. Ligonzo, R. Avolio, A. Napolitano and M. d'Ischia, Adv. Funct. Mater., 2014, 24, 7161-7172.

10 J. H. Ryu, P. B. Messersmith and H. Lee, ACS Appl. Mater. Interfaces, 2018, 10, 7523-7540.

11 Y. Liu, K. Ai and L. Lu, Chem. Rev., 2014, 114, 5057-5115.

12 H. Lee, S. M. Dellatore, W. M. Miller and P. B. Messersmith, Science, 2007, 318, 426-430.

13 X. Zhong, K. Yang, Z. Dong, X. Yi, Y. Wang, C. Ge, Y. Zhao and Z. Liu, Adv. Funct. Mater., 2015, 25, 7327-7336.
14 Y. Tokura, S. Harvey, C. Chen, Y. Wu, D. Y. W. Ng and T. Weil, Angew. Chem., Int. Ed., 2018, 57, 1587-1591.

15 J. Zhou, C. Wang, P. Wang, P. B. Messersmith and H. Duan, Chem. Mater., 2015, 27, 3071-3076.

16 M. d'Ischia, A. Napolitano, V. Ball, C.-T. Chen and M. J. Buehler, Acc. Chem. Res., 2014, 47, 3541-3550.

17 K. M. Im, T.-W. Kim and J.-R. Jeon, ACS Biomater. Sci. Eng., 2017, 3, 628-636.

18 Z. F. Gao, X. Y. Wang, J. B. Gao and F. Xia, $R S C A d v .$, 2019, 9, 20492-20496.

19 F. Ponzio, J. Barthes, J. Bour, M. Michel, P. Bertani, J. Hemmerle, M. d'Ischia and V. Ball, Chem. Mater., 2016, 28, 4697-4705.

20 H. Yin, K. Zhang, L. Wang, K. Zhou, J. Zeng, D. Gao, Z. Xia and Q. Fu, Nanoscale, 2018, 10, 18064-18073.

21 Q. Wei, F. Zhang, J. Li, B. Li and C. Zhao, Polym. Chem., 2010, 1, 1430-1433.

22 X. Li, H. Shan, M. Cao and B. Li, J. Membr. Sci., 2018, 555, 237-249.

23 M. S. H. Akash and K. Rehman, J. Controlled Release, 2015, 209, 120-138.

24 M. F. Dario, A. R. Baby and M. V. R. Velasco, J. Photochem. Photobiol., B, 2015, 153, 240-246.

25 M. Brenner and V. J. Hearing, Photochem. Photobiol., 2008, 84, 539-549.

26 S. M. Kang, N. S. Hwang, J. Yeom, S. Y. Park, P. B. Messersmith, I. S. Choi, R. Langer, D. G. Anderson and H. Lee, Adv. Funct. Mater., 2012, 22, 2949-2955.

27 Q. Fu, X. Li, Q. Zhang, F. Yang, W. Wei and Z. Xia, J. Chromatogr. A, 2015, 1416, 94-102.

28 X. Zhang, M. Liu, B. Yang, X. Zhang and Y. Wei, Colloids Surf., B, 2013, 112, 81-86.

29 J. Y. Kim, B. S. Lee, J. Choi, B. J. Kim, J. Y. Choi, S. M. Kang, S. H. Yang and I. S. Choi, Angew. Chem., Int. Ed., 2016, 55, 15306-15309.

30 Q. Wu, M. Niu, X. Chen, L. Tan, C. Fu, X. Ren, J. Ren, L. Li, K. Xu, H. Zhong and X. Meng, Biomaterials, 2018, 162, 132-143.

31 M. R. Prausnitz, S. Mitragotri and R. Langer, Nat. Rev. Drug Discovery, 2004, 3, 115.

32 M. B. Brown, G. P. Martin, S. A. Jones and F. K. Akomeah, Drug Delivery, 2006, 13, 175-187.

33 M. Liu, G. Zeng, K. Wang, Q. Wan, L. Tao, X. Zhang and Y. Wei, Nanoscale, 2016, 8, 16819-16840.

34 X. Fang, J. Zhao, K. Zhang, P. Yang, L. Qiao and B. Liu, ACS Appl. Mater. Interfaces, 2016, 8, 6363-6370.

35 E. N. Jayaweera, K. R. Wijewardhana, T. K. Ekanayaka, A. Shahzad and J.-K. Song, ACS Sustainable Chem. Eng., 2018, 6, 6321-6327.

36 H. Li, Y. Gong, C. Fu, H. Zhou, W. Yang, M. Guo, M. Li and Y. Kuang, J. Mater. Chem. A, 2017, 5, 3875-3887.

37 W. Yuan, Q. Zhou, Y. Li and G. Shi, Nanoscale, 2015, 7, 16361-16365.

38 M. Zhang, C. Wang, Q. Wang, M. Jian and Y. Zhang, ACS Appl. Mater. Interfaces, 2016, 8, 20894-20899. 